\title{
Ökonomische und politische Prinzipien im Umbruch: die „Quantitative Revolution“ der Geographie als Spiegel bürrgerlichen Bewusstseins
}

\author{
Ulrich Eisel \\ ehemals: Kulturgeschichte der Natur, Fachbereich 14 Landschaftsentwicklung, Technische Universität Berlin, \\ Berlin, Germany \\ Correspondence to: Ulrich Eisel (mail@ueisel.de)
}

Received: 10 November 2016 - Accepted: 22 November 2016 - Published: 30 January 2017

\begin{abstract}
Kurzfassung. The quantitive revolution in geography was the methodological expression of a shift in paradigm. Nomological thinking took over from the idiographic approach of classic geography. The classic paradigm had been that of a desirable identity of concrete working, active humans with their concrete natural surroundings: landscape was imagined as Lebensraum. The logic of industrial production processes contrasts with this; it creates an identity of scientifically analysed human work sequences with machines, and it thus represents a form of adapting to nature by abstracting holistically integrated ways of carrying out work. The geographical paradigm had no theoretical tools with which to approach this relationship between humans and nature. With regard to the theoretical ideas underlying it, this methodological change corresponds, on the one hand, to the transition from following a humanist concept of the individual, which guides idiographic thinking, to using a democratic concept of the individual, which correlates with the principles of experiment-based empirical sciences. On the other hand, geography's move towards an abstract concept of space reflects the degree to which industrial production methods are abstracted. The "spatial approach", the "behavioural approach", and "humanistic geography" are interpreted and contrasted with the idiographic paradigm within this coordinate system.
\end{abstract}

Als ich die freundliche Einladung ${ }^{1}$ erhielt, zur Begründung der Ursachen der „Quantitativen Revolution“ ”etwas beizutragen, habe ich sehr gezögert. Ich war nicht begeistert von der Vorstellung, meine Erklärungen von vor 35 Jahren (Eisel, 1980) abermals zu wiederholen, habe mich dann aber doch umstimmen lassen. Natürlich werde ich dieses Grundgerüst aus heutiger Perspektive ergänzen; es erhält letztlich dadurch eine etwas andere Stoßrichtung.

Sodann werde ich zunächst einleitend versuchen, meine Art des Zugangs zu theoretischer Arbeit überhaupt, damit also auch zur Untersuchung der quantitativen Revolution in

\footnotetext{
${ }^{1}$ Der Text basiert auf einem Vortrag anlässlich des Workshops „Raum.Gesetze.Daten. Zur Geschichte der quantitativen Revolution in der deutschsprachigen Geographie“" im November 2015 an der Universität Erlangen. Er wurde in der Diktion des gesprochenen Wortes sowie eines komprimierten Überblicks belassen und nur um einige Präzisierungen und Belege ergänzt.
}

der Geographie, zu schildern. In der Vergangenheit hat meine Art von Metatheorie immer wieder zu Fragezeichen geführt. Mich stört das nicht, aber kultivieren muss man diese Verunsicherung auch nicht.

\section{Credo}

Mein Ausgangspunkt ist die folgende These: „Das gesellschaftliche Sein bestimmt das Bewusstsein“. Das ist das Credo des Historischen Materialismus und eine grundlegende Absage an jede Art von Konstruktivismus noch bevor es diesen gab und an Ontologie; damals hießen die Gegner noch Idealismus und mechanischer Materialismus. ${ }^{2}$ Mit dem be-

\footnotetext{
${ }^{2}$ Mit diesem Credo ist nicht zwingend eine abbildtheoretische Position verbunden. Die Pointe besteht darin, dass ein eigenständig funktionierendes Universum von Ideen in seiner Konstitutionsfunktion für diejenigen realen Prozesse, deren erscheinendes Bewusst-
} 
rühmten Satz von Marx hängt der Topos zusammen, dass Wissen, Denken, jede Theorie sogenanntes erscheinendes Bewusstsein ist. Die Formulierung suggeriert, dass irgendein spezifisches Denken ,,auftaucht“ in einer spezifischen historischen Situation. Das Denken reflektiert etwas Wirkliches de facto, gewissermaßen hinter dem Rücken des Theoretikers, ganz egal, ob der das weiß und auch so gewollt und gemeint hat oder nicht. Das ähnelt dem Phänomen Zeitgeist, ist aber anders begründet.

Ein Beispiel wäre Darwins Evolutionstheorie. Sie übersetzt den Malthusianismus in eine Entwicklungstheorie der Natur, und beide Theorien sind das erscheinende Bewusstsein des Konkurrenzkapitalismus. Das wird allgemein so gesehen, und in diesem Fall hat auch Darwin selbst diese Verbindung zu Malthus gezogen. Darwin hat sich dem ökonomietheoretischen Zeitgeist angeschlossen und der war erscheinendes Bewusstsein eines spezifischen Aspekts des Kapitalismus.

Das Beispiel zeigt, dass man die Ebene von Wirklichkeit benennen muss, die da erscheint, wenn man von erscheinendem Bewusstsein redet. Es könnte z.B. auch gemeint sein, dass das Elternhaus durch Erziehung das Bewusstsein des Kindes prägt, so dass in diesem Bewusstsein später das gesellschaftliche Sein, wie es sich im Elternhaus ereignete, tradiert und erkennbar wird. Das ist aber nicht gemeint. Mit so etwas werden wir in Tatort-Filmen traktiert, in denen der erwachsene Sohn des Industriellen ein Leben lang unter der strengen Erziehung und Missachtung seines gnadenlos erfolgreichen Vaters zerbricht. Die Tochter bringt dann den Vater um und die Mutter wird noch depressiver, als sie schon war.

Mit gesellschaftlichem Sein sind demgegenüber die Produktionsweise und die Produktionsverhältnisse gemeint, sowie die Klassenposition eines Theoretikers. Zum Beispiel ist dann Ricardo das erscheinende Bewusstsein der industriekapitalistischen Produktionsweise aus der Perspektive der Grundbesitzer. Er hatte die Funktionsweise der Mehrwertbildung für das Kapital erkannt, aber mit einem spezifischen Begriff von Arbeit als konkreter Arbeit die Agrarproduktion als mehrwertbildende hervorgehoben - und sich damit die Kritik von Marx eingehandelt.

Mit gesellschaftlichem Sein sind also nicht so etwas wie Lebenssituationen gemeint, sondern Strukturen ökonomischer Beziehungen in historischer Perspektive. Alltagsweltlich gesehen ist diese Ebene abstrakt, gesellschaftstheoretisch gesehen sind das konkrete Überlebensbedingungen von Gesellschaften.

sein diese Ideen sind, gleichberechtigt berücksichtigt werden muss. Dialektisches Denken ist nicht einfach zu haben; es muss Widersprüche fruchtbar machen. (Zur komplexen epistemologischen Rekonstruktion dieses Denkens vgl. Althusser et al., 2015 sowie zu einer Art Entschuldigung für gewisse materialistische Vereinfachungen Engels, 1966.)
Ich beende jetzt diese Skizze der allgemeinen Voraussetzungen, die ich seinerzeit auf die Geographie angewandt habe.

\section{Individualität I}

In der Geographie galt es einen Paradigmenwechsel zu erklären. Paradigmen sind fundamentale Grundmuster erscheinenden Bewusstseins. Demzufolge musste die Landschaftsund Länderkunde als erscheinendes Bewusstsein einer Produktionsweise und Klassenposition begriffen werden. Dasselbe galt für die moderne Geographie. Ausgangspunkt für die Charakterisierung des klassischen Paradigmas war natürlich das, was die Geographen selbst darüber sagten. Das ist allerlei, der Kern besteht darin, dass sie die Geographie gegenüber den sogenannten nomothetischen Gesetzeswissenschaften als idiographische Wissenschaft bezeichneten. ${ }^{3}$ Das bedeutet: Es gilt immer, die besondere Eigenart eines Gegenstandes zu verstehen. Das leitet sich davon ab, dass der Gegenstand als Individuum begriffen wird. Das heißt: Das Prinzip des Allgemeinen, also das Ergebnis von Verallgemeinerungen, besteht nicht in einem Gesetz über ein einzelnes Ereignis, sondern in der Individualität dieses Ereignisses. Das Einzelne wird nicht formal unter ein Gesetz subsummiert, um es zu erklären, sondern es ist besonderer Ausdruck eines allgemeinen Gesetzes/Maßstabs/Prinzips oder Wertes. Es ist „typisch“. Das gilt in beide Richtungen: Einzelnes ist typisch für ein allgemeines Prinzip; und es ist typisch dafür, wie es unverwechselbar individuell das allgemeine Prinzip repräsentiert. Stellen Sie sich als Beispiel Charlie Chaplin vor: Der ist auf wunderbare Weise typisch für des allgemeine Prinzip der Komik, aber er ist zugleich unverwechselbar typisch für seine individuelle Art der Komik. Das ist jener besondere Ausdruck des Allgemeinen, von dem ich gesprochen habe. Demgegenüber gibt es im nomothetischen Denken der ,Gesetzeswissenschaften“ so etwas wie ein Ausdrucksgeschehen überhaupt nicht. Das einzelne Ereignis ist nicht eine Inkarnation, welche Allgemeines enthält und gewissermaßen auf seine besondere Weise vorzeigt.

Diese idiographische Konstruktion und Idee von Allgemeingültigkeit folgt aus dem humanistischen Begriff des Individuums. Er entsteht in der Antike und wird vom Christentum überformt und tradiert bis heute. Dieser Begriff von Individualität und damit von Allgemeinheit ist derjenige, der das Paradigma der klassischen Geographie bestimmt hat. In Kurzform noch einmal das Vorherige auf einer anderen Ebene: Individualität bedeutet Unteilbarkeit. Eine Einheit bilden sollen Leib und Seele bzw. Geist, Freiheit und Gesetz des Handelns, also Wille und Vernunft; dem entspricht die Einheit von Möglichkeit und Notwendigkeit. Diese Relationen sind eine Einheit, nämlich Unteilbarkeit, unter der Perspektive, dass sie alle aus Anpassungsbeziehungen zwischen geg-

\footnotetext{
${ }^{3} \mathrm{Zu}$ den beiden Begriffen vgl. Windelband (1924).
} 
nerischen Polen bestehen; die Einheit ist dann eigentlich eine Paradoxie - die der Individualität.

Das Paradox löst sich auf, wenn die Beziehungen als Entwicklungsprozess begriffen werden in einem Anpassungsverhältnis zwischen Innen und Außen. Der Ort der Freiheit, des Willens, der Seele, des Geistes, der Möglichkeiten ist innen. Der Leib ist das umgebende Außen, mit Naturgesetzen und Notwendigkeiten. Das Denkmuster ist übertragbar. In der Geographie betraf es die Freiheit des Menschen bzw. der Völker, die sich an die Natur und deren Gesetze anpassen; sie repräsentieren die Seite „Geist“". Nimmt man also den Menschen als ganzen, dann steht dieses Individuum als vernünftig frei handelndes der Vorsehung Gottes - in der Moderne den Gesetzen der Natur - gegenüber. Dann ist die Natur die Seite der äußeren Notwendigkeiten - zumindest in geographischer Perspektive, denn gesellschaftstheoretisch ist zunächst das gesellschaftliche System die Seite der äußeren Notwendigkeiten; das wäre dann eine soziologische oder aber politische Perspektive.

Wenn diese Relation zwischen Mensch und Natur sich so gestaltet, dass sie wie Individualität funktioniert, dann wird aus dem Paradox eine Entwicklungseinheit. Das geht im Ursprungsmodell so: Der Mensch entwickelt sich als Individuum, wenn er seine natürlichen Anlagen, die gewissermaßen seine Lebenskräfte darstellen, immer im Abgleich mit objektiven Wertmaßstäben zur Vervollkommnung seiner Persönlichkeit nutzt. Er besiegt mit gutem Willen die schlechten Anlagen; dann kann er mit Vernunft die objektiven Werte mit den guten Anlagen für sich fruchtbar machen. Er versucht ein besonderes Exemplar dessen zu werden, was den objektiven Maßstäben als Idealbild entnommen werden kann. Er befolgt diese Maßstäbe nicht einfach mechanisch als Gesetze, sondern versucht auch für sich das Beste aus ihnen zu machen. Sie erinnern sich an die doppelte Ausrichtung des Typisch-seins bei Charlie Chaplin: typisch für das Allgemeine und für sich selbst. So lebt dieser Mensch seine Freiheit aus. Freiheit ist das, weil er sich nicht mechanisch Gesetzen unterwirft, sondern auf besondere Weise sich selbst zur Geltung und damit zu Allgemeingültigkeit bringt.

Ein solcher Mensch entwickelt Eigenart, hat Charakter, ist eine Persönlichkeit. Ich erzähle Ihnen hier nichts Neues, sie alle arbeiten täglich daran. Ein Mensch ohne Eigenart, Charakter (selbst schlechten), Persönlichkeit usw. ist keiner, eine Null. Misslungen wäre dann die Bindung der Anlagen an objektive Werte. Wenn ein Einzelner nichts Besonderes ist, dann fehlt ihm etwas, das ihm objektiv Geltung verleiht. Dann aber hat er nichts, was ihn individuell heraushebt. Man sieht: Es bleibt bei der Paradoxie; aber im Entwicklungsgeschehen einer Anpassung des einen Pols an den anderen $\mathrm{zu}$ einer gelingenden Einheit wird sie zum erfüllten Leben, offenbar weil die Paradoxie irgendwie produktiv genutzt wird.

\section{Land versus Fallgesetz}

Dieses Denkmuster ist die mentale Grundlage des Abendlandes. Es ist übertragbar und durchzieht alle Gegenstandsbereiche unserer Kultur. Im Falle der klassischen Geographie ist dieses Individuum das Land. ${ }^{4}$ Länderkunde ist die Aufgabe der Geographie. Die war als Paradigma ganz praktisch in den Reisebeschreibungen der Entdecker entstanden. Somit galt es, die Länder in ihrer Einmaligkeit als Einheit zweier Pole, die sich in einem Anpassungsverhältnis befinden, zu beschreiben. Dazu musste man die Eigenart ihrer Landschaften in Verbindung mit der Eigenart der Bewohner als historischen Entwicklungsprozess beschreiben. Landschaften dagegen waren keine Individuen; sie galten als ganzheitliche Modelle von Eigenart, d.h., auch sie wurden gemäß der Idee des Individuums gedacht, andernfalls hätte man in der Länderkunde nichts mit ihnen anfangen können. Aber sie standen für das allgemeine Prinzip des Typisch-seins, nicht für die einzelne historische Lösung; sie sind gewissermaßen Strukturbeispiele von Individualität. Damit war die Landschaftskunde so etwas wie eine idiographische Modelltheorie. Gesetzescharakter hatte das Besondere des erdräumlichen ganzheitlichen Ereignisses. Das ist das Gegenteil vom Typus des Denkens, welches das Fallgesetz hervorgebracht hat.

Dieses Paradigma war mit den experimentellen Erfahrungswissenschaften inkompatibel - auch und vor allem in der Physischen Geographie, obwohl die ja doch als $\mathrm{Na}-$ turwissenschaft begriffen wurde. Deshalb bestand der $\mathrm{Pa}$ radigmenwechsel in einer sogenannten quantitativen Revolution, in der das idiographische Denken durch das experimentalwissenschaftliche ersetzt wurde. Denn Quantifizierung von empirischen Beobachtungen in der Weise, dass nicht nur in sogenannter Feldforschung gezählt und gemessen wird, sondern von den alltagsweltlichen Randbedingungen dadurch abstrahiert wird, dass Theorien mathematisch formalisiert werden und standardisierte Experimente eigens ersonnen und durchgeführt werden, verlässt den Horizont des idiographischen Denkens; es widerspricht ihm.

Damit wurde auch der im Untergrund wirksame Individualitätsbegriff ausgewechselt; darauf komme ich noch zu sprechen. Quantifizierung stand und steht für intersubjektive Verifikationsmöglichkeiten. Dem stand im klassischen Paradigma ein intuitives Verständnis der landschaftlichen Sachverhalte gegenüber (vgl. auch hierzu Stewig, 1979:172-185). Gemeint war - wenn man es sehr gutwillig interpretiert ungefähr das, was wir Hermeneutik nennen würden; Martin Schwind spricht von der „Kulturlandschaft als objektivierte(m) Geist" (Schwind, 1951). Oft wurde auch auf die Fähigkeit zu professionell-gefühlvoller Schau verwiesen. Gemeint war also die sensible Sicht auf sinnvolle Zusammenhänge ei-

\footnotetext{
${ }^{4}$ Vgl. - ungeachtet einiger immanenter Ungereimtheiten Kraus (1979), sowie insgesamt Stewig (1979), dort insbesondere den Anhang zur „Bestandsaufnahme zur Situation der deutschen Schul- und Hochschulgeographie“:172-185.
} 
nes Ausdrucksgeschehens, in dem sich das Mensch-NaturVerhältnis als jeweils besonderes Aneignungsverhältnis spiegelt. Die Erde ist die Überlebensgrundlage, und die Völker verwirklichen ihre Eigenart, ihren Volkscharakter, wie Herder es genannt hatte, in einem historischen Prozess der Anpassung. So entstehen Länder.

Das Mensch-Natur-Verhältnis in geschichtlicher Perspektive war aber auch das Programm der Geschichtsphilosophie überhaupt. Wie unterschied sich die Geographie davon? Die Geschichtsphilosophie suchte Antworten unter der Perspektive, dass und wie das Wesen des Subjekts, bestehend aus Vernunft und freiem Willen, historische Entwicklung verursacht. Demgegenüber fragte die Geographie danach, wie die Natur als Gegenspieler und Material von Vernunft und Willen Entwicklung beeinflusst. Damit hatte sie eine doppelte Perspektive, sie setzte zwei Determinationsrichtungen voraus: Die Natur hat Einfluss auf die kulturellen Entwicklungsmöglichkeiten und menschlicher Wille sowie die Vernunft des Menschen gleichfalls. Die Geographie musste folglich in ihrer Fachgeschichte eine Paradoxie aushalten. Das ist experimentalwissenschaftlich unmöglich. Diese exklusive Sichtweise im Rahmen der Positivierung der Geschichtsphilosophie, ein Prozess, der auch alle anderen modernen Gesellschaftswissenschaften entstehen ließ, folgte einfach der Praxis des Paradigmas: den Reisebeschreibungen. Das Fach betrieb Erd-kunde, es kündete von dem, was vom Planeten Erde aus gesehen dazu führt, dass Landschaften so aussehen wie sie aussehen, Völker so überleben, wie sie überleben und moderne Gesellschaften sich durch Grenzen als Staaten definieren. Hätten die Geographen die Erde weggelassen, wären sie Völkerkundler, Soziologen, Ökonomen, Politologen usw. geworden. Deshalb formulierten sie das Abgrenzungskriterium „Raum“ gegenüber dem, was sie „systematische“ Wissenschaft nannten.

\section{Ein kurzer Blick zurück}

Dieses Abgrenzungskriterium war Ende der 60er Jahre unser Ausgangspunkt der theoretischen Fachkritik gewesen. ${ }^{5}$ Wir stellten fest, dass mit der Zauberformel ,räumliche Wissenschaft" alles das vermieden wurde, was wir für unerlässlich hielten, wenn man vernünftige zeitgemäße, vor allem aber kritische Gesellschaftswissenschaft betreiben wollte (vgl. exemplarisch die Kritik von Werlen, 1987, 1997). Das nämlich wurde von den Geographen in die systematische Wissenschaft verwiesen. Für die Physische Geographie galt das Gleiche im Verhältnis zur Naturwissenschaft. Vor allem der naive, sensualistische Empirismus, der mit der Fachbegründung vom Objekt her, von der Landschaft als Ganzheit und dem Land als Individuum her, verbunden war, war wissenschaftstheoretisch völlig anachronistisch. Gerhard Hard vor allem hat diesen Sensualismus in vielen Schriften mit beißendem Spott überzogen; so hat er ihn beispielsweise einen

\footnotetext{
${ }^{5}$ Vgl. die Hefte des Geografiker 3-7/8.
}

„extrem theorieabstinente(n) Hyperpositivismus des gesunden Menschenverstandes" (Hard, 1977:143) genannt. ${ }^{6}$

Mir genügten der Spott - obwohl ich große Freude daran hatte - und die sprachanalytische Kritik nicht. Meine These war: Die Geographen können nicht 200 Jahre lang verrückt gewesen sein mit ihrer ärmlichen Theorie und Wissenschaftstheorie. Was also verbirgt sich hinter diesem Abgrenzungskriterium „Raum“ bzw. ,räumlich“ und wird, so wie Paradigmen nun mal wirken, auf Gedeih und Verderb verteidigt? Ich nahm, im Gegensatz zu den anderen Kritikern, die Geographie ernst, trotz meiner eigenen wissenschaftstheoretischen und politischen Kritik. Denn gegenüber dem Vorwurf der gesellschaftlichen Bedeutungslosigkeit als moderne Wissenschaft, vertrat ich die These, dass ein Fach nicht 200 Jahre bedeutungslos überleben kann. Die Frage war nur: Welchem Herren dient sie?

Damit sind wir zu meinen einleitenden Bemerkungen zurückgekehrt. Ich fragte mich nach dem ,erscheinenden Bewusstsein" einer Produktionsweise und von Produktionsverhältnissen. Das hieß, die Tatsache, dass ich die Geographie fortan nicht verhöhnte, sondern zu verstehen suchte, folgte aus einer marxistischen Fundamentalbedingung theoretischen Denkens. Die anderen Marxisten hatten Mühe damit; sie begriffen politische Kritik und die Frage nach dem Herren, dem die Geographie dient, nicht als Nachweis des gesellschaftlichen Ursprungs, sondern der politischen Folgen dieser Raumwissenschaft - vor allem in der nationalsozialistischen Ostkolonisation und überhaupt im Kolonialismus. Ich nenne das Entlarvungsmarxismus. Er ist politisch respektabel, versteht aber von seinem Gegenstand, in diesem Falle der Geographie, nichts; der theoretische Gewinn ist gering. Eine Theorie oder Metatheorie der Geographie entsteht dabei nicht. Die theoretischen Wege der linken Kritiker der Geographie trennten sich, die lebenspraktischen nicht. ${ }^{7}$

\section{Erdraum: der äußere Standpunkt in der Geschichte}

Dass der Begriff Raum eine Signatur dafür ist, von der Natur her geschichtliche Entwicklung zu denken, sagten die Geographen selbst; das sollte auch für die Entwicklung der modernen Gesellschaft und Staaten gelten. Das Mensch-NaturVerhältnis der modernen Gesellschaft besteht in der industriekapitalistischen Produktionsweise. Wie näherte sich die Geographie diesem Verhältnis? Sie formulierte ihren Ausgangspunkt grundsätzlich ja als einen äußeren Standpunkt Ratzel z.B. hat ihn so charakterisiert: „Wir sehen den Geist von aussen her, indem wir selber unsern Standpunkt in der

\footnotetext{
${ }^{6} \mathrm{Vgl}$. auch exemplarisch mit zwei ganz unterschiedlichen Stoßrichtungen Hard (1970) sowie 2002 mit anschließender Diskussion zwischen Wirth (1972) und Hard (1972) in Geografiker 7/8.

${ }^{7} \mathrm{Zu}$ einer Selbstreflexion meiner Position im Sinne eines ,Werdeganges" sowie zu meinem Umgang mit dem Raumbegriff in der Geographie vgl. Eisel (2009a).
} 
umgebenden Natur nehmen“ (Ratzel, 1882:385) (vgl. auch Schlüter, 1906:592 f.). Das war die Übersetzung von ,räumlich“ in die Wahrnehmungsebene, die mit jener der als systematische apostrophierten Wissenschaften verglichen werden konnte. Denen wurde der ,innere Standpunkt“ zugeordnet. Das heißt, die Geographie interessierte sich dafür, was man vom Industriekapitalismus von außen sehen kann; dessen Inneres als Mensch-Natur-Verhältnis interessierte nicht, obwohl sie ja das Mensch-Natur-Verhältnis untersuchte. Das Abgrenzungskriterium ,,räumlich“ blockierte in der Geographie gewissermaßen einen angemessenen Begriff von Industrie.

Der Blick von außen war der landschaftliche Blick. Landschaft war eine Formel für die gelingende Verbindung von Völkern mit ihren Naturgrundlagen in einer Einheit, d.h. dafür, dass die Erde sich in Lebensräume aufteilt und so klassifizieren lässt. Länder hingegen warfen das sogenannte Grenzproblem auf. Das bestand darin, dass politische Staatsgrenzen und naturräumliche Grenzen nicht übereinstimmten. Das wurde im Grunde beklagt und endlos diskutiert, zuletzt aber dann meist irgendwie doch als in modernen Zeiten unvermeidlich anerkannt; hier soll uns nicht beschäftigen mit welchen theoretischen Schachzügen.

Die Pointe des lebensräumlichen Denkens im Hinblick auf den Schutz vor der industriellen Produktionsweise ist, dass die Vorstellung von geschichtlicher Entwicklung durch $\mathrm{Na}$ turanpassung durch das klassische Paradigma einer Idee von konkreter Natur und vom konkret arbeitenden und handelnden Menschen unterlag. Das war eine Situation, die man vorfindet, wenn man im Kongo-Becken auf Entdeckungsfahrt geht und Pygmäen beobachtet. Aber in modernen Zivilisationen warf das Probleme auf. Wie der Mensch sich konkrete Umwelt aneignet, ist in einer Maschinenfabrik oder Textilfabrik nicht mehr ohne weiteres landschaftlich sichtbar; der Produktionsprozess taucht in der Landschaft nicht auf: ,(N)ur an wenigen Punkten übt die Industrie eine echte landschaftsgestaltende Wirkung aus. Hinter niedrigen Fabrikmauern, kaum sichtbar im Raum, werden Güter für Millionen von Menschen hergestellt" (Otremba, 1960:243). Trotzdem: Ein Paradigma ist ein Paradigma, ist ein Paradigma, ist ein Paradigma, ... . . Das geographische ist das Paradigma der Einheit der Welt in konkreter Natur - eben Erdkunde.

\section{Das Innere des industriellen Mensch-Natur-Verhältnisses}

Die industrielle Produktionsweise ist demgegenüber die Praxis der Einheit in abstrakter Natur. Dort wird an Fließbändern mit einem Kalkül der Zerteilung eines Produkts in mechanisch zusammensetzbare Einzelteile sowie der Aufteilung menschlicher Arbeitstätigkeit gemäß einem Kalkül der Ausnutzung und Optimierung der Sequenzen von mechanischer Kraftausübung das Mensch-Natur-Verhältnis konstituiert. In einem Lehrbuch der Ergonomie lautet das so: „(D)ie Ergo- nomie versucht (...) die Variabilität des Menschen (oder der Menschen) als Teilsystem in Arbeitssystemen zu analysieren und $\mathrm{zu}$ beschreiben. Die Ergonomie untersucht deshalb das statische und dynamische Verhalten der Menschen unter Arbeitsbedingungen. Ziel der Untersuchungen ist die optimale Anpassung zwischen Mensch und Arbeit. Diese Optimierung von Arbeitssystemen kann dabei sowohl durch Anpassung an den Menschen, wie auch durch Anpassung der Menschen an die Arbeit geschehen. Schlechte Anpassung hat eine verringerte Leistungsfähigkeit des Arbeitssystems zur Folge“ (Laurig und Rohmert, 1974:113; Zitat gram. verändert; vgl. auch Abb. 1). Was verbirgt sich hinter dieser Definition? Sobald nach der ,zweiten ursprünglichen Akkumulation“ von Kapital das ökonomische Subjekt „Kapital“ sich der Produktion bemächtigt, stellt sich die Welt auf den Kopf: Nicht die Menschen leisten Arbeit, sondern Arbeit ist eine Systemleistung, in der die Menschen als Teilsysteme mit den Maschinen koordiniert werden. Die Kapitalform ist die Einheit eines Systems, das aus konstantem und variablem Kapital besteht. Arbeit wird als konstantes Kapital maschinell und als variables Kapital an die Maschinen angehängt.

Das Ziel der Anpassung besteht darin, dass relativer Mehrwert durch technische Rationalisierung gesteigert werden kann. In der Konkurrenz der Kapitale ist dieses Bestreben zwingend. So kann durch die Übersetzung von Theorien der Mechanik aus der Physik in Technik, also naturwissenschaftlich und ingenieurwissenschaftlich, eine Wirkung auf der Wertebene, also in der realen Ökonomie, erzeugt werden, weil Arbeit sowohl eine Kraft ist, als auch - durch den Lohn - ein Warenwert. Je technisch geschickter sie ausgebeutet wird, umso rentabler ist ihr Einsatz als variables Kapital. ${ }^{8}$

So sieht der Blick von Innen auf den Kapitalismus aus. Die Kapitalisten nennen das Fortschritt durch Rationalisierung. Dieser Blick widerspricht der Untersuchung konkreter Lebenswelten und Lebensräume so, wie das idiographische Denken, mit seiner Suche nach dem Besonderen, dem Fallgesetz widerspricht. Denn alles ist in der industriellen Produktionsweise auf abstrakte Verallgemeinerung, auf Gleichmacherei, ausgerichtet: bei den Produkten und hinsichtlich der individuellen Fähigkeiten in den Arbeitsprozessen. Das Besondere zählt nicht - ebenso wenig wie beim Fallgesetz der Unterschied zwischen Feder und Bleiglotz.

Die daraus entstehende Welt wurde von den Geographen zur Kenntnis genommen - was blieb ihnen auch übrig -, meist hinsichtlich der landschaftlichen Folgen beklagt (sie hatten ja jenen ,äußeren Standpunkt“"), aber insgesamt anerkannt, jedoch mit der Einschränkung, dass es der beklagten negativen Folgen durch den richtigen Einsatz der neuen technischen Möglichkeiten der Naturbeherrschung vorzubeugen gelte. Die industrielle Produktionsweise wurde als arrivier-

\footnotetext{
${ }^{8}$ In der Kybernetik, Informatik und Forschung zur künstlichen Intelligenz wird nun in einem weiteren Schritt der Industrialisierung, neben der Handarbeit, die Kopfarbeit der Kapitalverwertung einverleibt.
} 


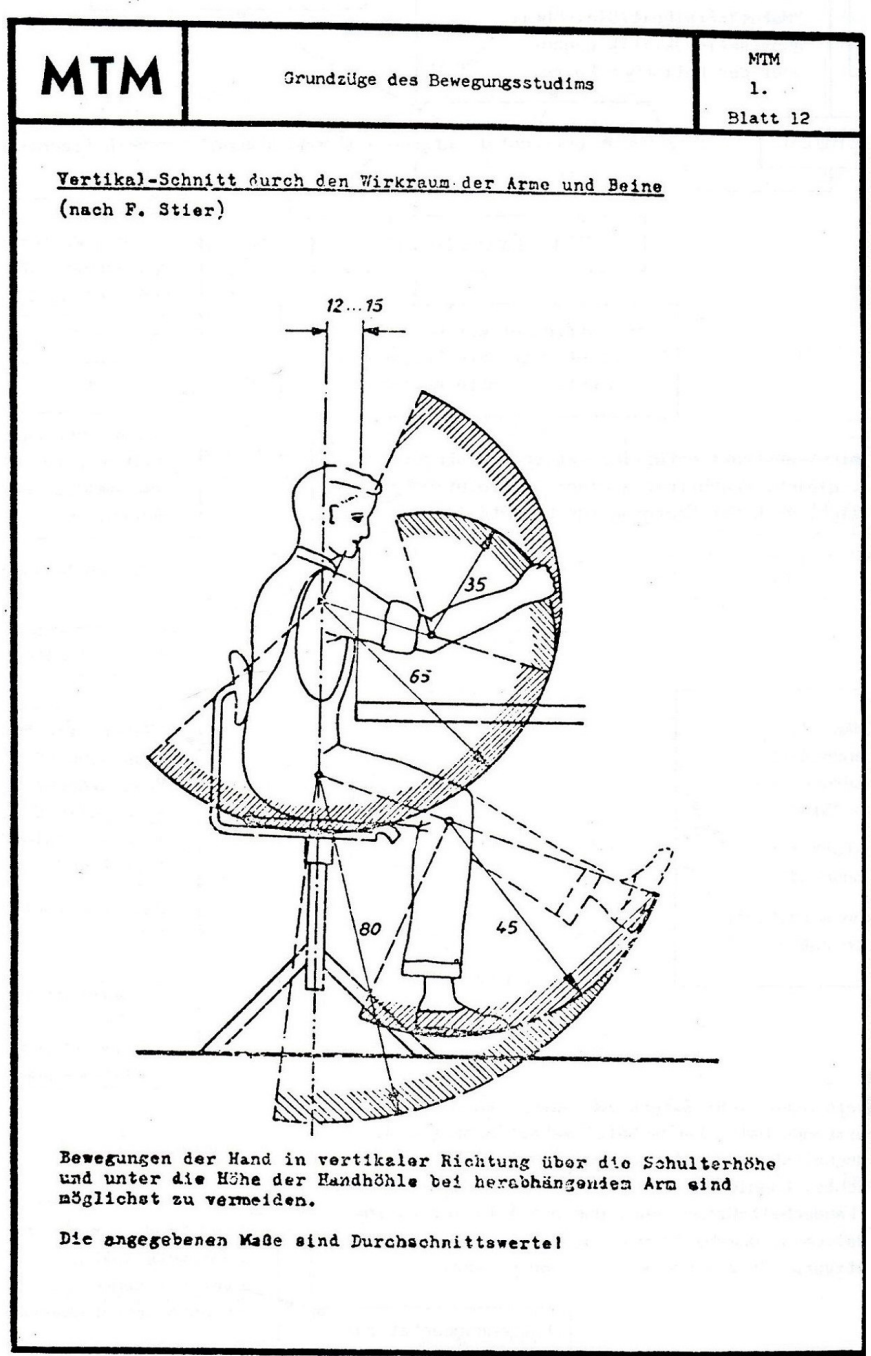

Abb. 1.

te Technik wie ein weiterentwickeltes Handwerk, d.h. ohne Rücksicht auf die Differenzen zwischen Handwerk, Manufaktur und Industrieproduktion, begriffen. Diese Technik müsse nur sinnvoll betrieben werden, sagten sie. Sinnvoll hieß in der Regel in der deutschen Geographie: der nordischen Rasse und dem deutschen Wesen gemäß. So war das paradigmatisch Unverdauliche fachpolitisch handhabbar, und die politische Karriere des Faches recht unausweichlich.

\section{Die geographische Revolution als „erscheinendes Bewusstsein“"}

Das Credo der „social physics“ ist die Einheit der Welt in abstrakter Natur. Die Schriften von J. Q. Steward und William Warntz demonstrieren das eindrücklich, und sie reflektieren auch auf diese Idee und Position (Stewart, 1950, 1953;
Fig. 1

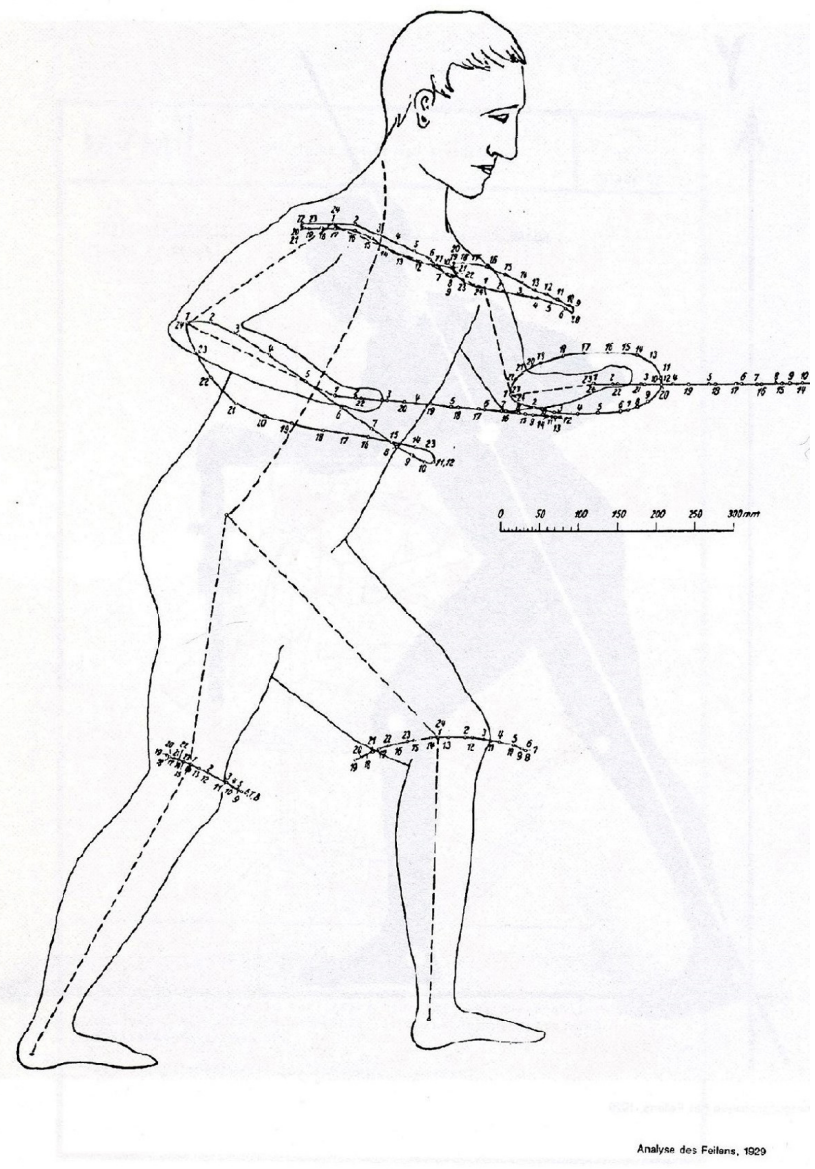

Quelle: RATIONALISIERUNG. Ausstellungskatalog zur Ausstellung in Berlin und Oberhausen im Jahre 1984, Staatliche Kunsthalle und NBGK,
S. 82 .

Stewart und Warntz, 1958, 1968; Warntz, 1957a, b, 1973). Die beiden gehörten zu den frühen Gallionsfiguren des sogenannten spatial approach ${ }^{9}$ in der sich anbahnenden Revolution. Wenn es also später in der Geographie einen Paradigmenwechsel gegeben haben sollte, dann hatte er stattgefunden, als Geographen sich der Sozialphysik verschrieben. Natürlich wurde - als das Paradigma ausgewechselt wurde - nicht der Vorgang als erscheinendes Bewusstsein im Hinblick auf den Unterschied zwischen vor-industrieller und industrieller Produktionsweise reflektiert, mit entsprechenden Sanierungsmaßnahmen im Sinn. Vielmehr musste der

\footnotetext{
${ }^{9}$ Zum spatial approch und dem nachfolgenden behavioral approach mit ausführlichen Literaturhinweisen vgl. das Kapitel „3. Die Oberfläche der anti-idiographischen Revolution“ in Eisel (1980:185-273); zum Untergrund der Oberfläche vgl. ebenda, Kapitel 5 und 6, beide in Teilen Nachgedruckt in Eisel (2009b).
} 
Raumbegriff transformiert werden: aus konkret wurde abstrakt, aus der Erde wurde eine Art angewandte Mathematik und angewandte Physik mit Raumbezug (vgl. Bunge, 1966). Denn trotz der Revolution gab es die Kontinuität des Abgrenzungskriteriums. Das Fach musste etwas Anderes machen, als die anderen Fächer. Es blieb beim ,äußeren Standpunkt“, aber Experimentalwissenschaft war nun möglich. Das hat die klassischen Geographen entsetzt (vgl. Dörrenhaus, 1971, dazu Hard, 1971).

Wenn nun eine vernünftige polit-ökonomische Erklärung der industriekapitalistischen Arbeitsteilung darin besteht, dass in der „großen Maschinerie“ der Fabrikarbeit, in der Taylorisierung, im Fordismus usw., die konkrete, einzelne „lebendige Arbeit" der Menschen in ihrem körperlich und geistig ganzheitlichen Verrichtungszusammenhang zu einer durch den Tauschwertproduktionsprozess hervorgerufenen abstrakten, aufgesplitterten Verrichtung entlang der Fließbänder wird, dann konnte und kann keine Perspektive weiter von diesem Kern der neuen Zeit entfernt sein als jene, die Naturanpassung als konkrete integrierte Leistung von Menschen in Lebensräumen von außen betrachten will. Was änderte sich durch den Bruch mit dem Lebensraum-Paradigma? Nach dem Paradigmenwechsel wird nun der Abstraktionsgrad der Arbeitsteilung im geographischen Denken wirksam. Damit werden die Phänomene in den dazu passenden Theoriebegriffen beschrieben: Erdkunde als Geometry, Theory of Movement, Nearness-Prinzip usw. (Bunge, 1966).

In einem zweiten Schritt wurde sogar dem neuen Paradigma immanent der Kampf angesagt. Im behavioral approach und cognitive behavioral approach wurde der Raumbegriff als Abgrenzungskriterium und Theoriespektrum aufgegeben. Wer etwas auf sich hielt, betrieb Verhaltensgeographie. An dieser Stelle war die deutsche klassische Geographie durch die Münchner Schule einigermaßen vorbereitet. Dort war im alten Paradigma ein etwas einfältiger sozialgeographischer Funktionalismus entwickelt worden, der formal ganz gut zu diesem zweiten Trend passte. Im behavioral approach wurde der Erdboden aus dem klassischen Paradigma ebenso verlassen wie auch die Abstraktion davon in räumlichen Standortmodellen im spatial approach. In der klassischen Geographie und im spatial approach ging es noch um objektive Raumbeziehungen; diese objektiven Raumbeziehungen wurden nun subjektiv. Die Abkehr vom inzwischen abstrakt gewordenen Raum wurde präzisiert formuliert im sogenannten cognitive behavioral approach - allen voran von David Harvey, bevor er mit Unterstützung von John Coltrane und den Beatles Marxist wurde. Raum war nichts mehr, was in der Welt objektiv gegeben war, sondern fand in den Köpfen der Menschen statt. Das, was dort stattfand, wurde als in Verhaltensgewohnheiten verfestigtes, inneres Bild von der Welt für diese genommen; in diesem mentalen Raum wird gehandelt so sahen es die modernen Geographen. Das Außen, auf das sich die Bilder in den Köpfen natürlich noch immer bezogen - die Menschen handelten ja nicht real im eigenen Inneren d.h., dass es einen solchen Bezug notwendigerweise gab und dass er zu einer komplizierteren Theoriebildung hätte führen müssen, wurde (und wird überwiegend bis heute) epistemologisch ignoriert.

Es gab nun eine Perzeptionsgeographie, eine Theorie der Mental Maps, eine Natural Hazards-Forschung usw. Allerdings sind die Revolutionsschritte vom objektiven abstrakten Raum zum subjektiven Raum nicht ganz so scharf zeitlich getrennt erfolgt, wie ich das jetzt schildere. Zum Beispiel haben Wolpert und Hägerstrand schon früh sogenannte Innovations- und Diffusionsforschung betrieben (exemplarisch: Wolpert, 1970; Hägerstrand, 1967); es ging darum, welches Verhalten zu der Diffusion von Neuerungen im Raum führt. Aber in ihrer Logik sind die Etappen so getrennt, wie sie dann auch schwerpunktmäßig zeitlich aufeinander folgten.

Mit der verhaltenstheoretischen Wendung war der Positivierungsprozess der Geschichtsphilosophie und der Naturrechtslehren nachvollzogen worden, der lange zuvor die anderen Sozialwissenschaften hervorgebracht hat als Handlungstheorie und Verhaltenstheorien in Soziologie, Psychologie, Ökonomie, Ethnologie. An der Ökonomie kann man das gut erläutern. Die vollzog einen Übergang von der objektiven Wertlehre zur subjektiven Wertlehre und in der Folge zur Preistheorie, Konsumtheorie, zu Präferenztheorien, usw. Das sogenannte Transformationsproblem war abgeschafft worden, nämlich die Frage, wie aus Arbeitswert Preise werden, d.h., wie der Übergang von der Produktionssphäre in die Zirkulationssphäre werttheoretisch zu begreifen sei. Entsprechend wurde beispielsweise in der ökonomischen Theorie vertreten, dass man die Wahl für neue Standorte einfach von der Wahrnehmung der Konsumenten her entscheiden solle. Im behavioral approach begriff man nun 50 Jahre später Erdräume als mentale Landkarten.

\section{Individualität II}

Vor diesem Hintergrund erwiesen sich die Revolutionsphasen des Paradigmenwechsels als (längst fällige) Annäherungen an die industriekapitalistische Produktionsweise: an deren Abstraktionsgrad der Verdinglichung von menschlichen Beziehungen und Tätigkeiten sowie an deren methodisches Ideal der Intersubjektivität - das ist das, was zur Kennzeichnung „Quantitative Revolution“ geführt hatte.

Der Aspekt der Intersubjektivität verweist aber auch auf den inhaltlichen Untergrund. Er setzt einen demokratischen Individualitätsbegriff voraus im Unterschied zu jenem humanistischen Individualitätsbegriff, der im idiographischen $\mathrm{Pa}$ radigma der klassischen Geographie wirksam gewesen war. Die Prinzipien demokratischer Herrschaft sind darauf ausgerichtet, personale Willkür zu unterbinden. Denn das war das Prinzip der feudalistischen Herrschaft gewesen: die Einheit von Souveränität und Person unter der Voraussetzung natürlicher Ungleichheit der Menschen. 
Wenn demgegenüber ein ganzes Volk Souverän sein soll, müssen Prinzipien und Verfahrensregeln gefunden werden, die ein Kollektiv zu einem praktikablen Entscheidungsträger machen. Diese Prinzipien und Regeln müssen andererseits mit den Grundrechten des Einzelnen konform gehen; aus denen werden sie abgeleitet. Der erstgenannte Aspekt begründet das Prinzip der Intersubjektivität. Es muss für jeden Einzelnen überprüfbar nachvollzogen werden können, ob das, worüber alle anderen reden, den Tatsachen entspricht. Deshalb müssen die Tatsachen eine Repräsentationsform haben, die sie von rein privaten Meinungen zu unterscheiden erlaubt. Intersubjektivität ist das formale Prinzip des Verhältnisses zwischen Einzelnem und gesellschaftlichem Ganzen im Hinblick auf den Zusammenhang der Einzelnen untereinander.

Der zweite Aspekt steuert gewissermaßen den Inhalt dieser formalen Beziehung. Uns interessiert unter diesen Inhalten das Gleichheitsprinzip. In diesem Prinzip wird die Gültigkeit von Tatbeständen so definiert, dass Individualität, d.h. der Einzelne in seiner Gültigkeit, formal gehandhabt werden kann. Das ist zwingend, da die überwundene Herrschaftsform gerade durch das Gegenteil charakterisiert war: Wenn Herrschaft personal fixiert ist, konstituiert die natürliche Stellung der Person ihre gesellschaftliche Geltung. Die ist je nach Position in einer Hierarchie der Beziehungen zur wichtigsten Person in einer Rangskala definiert. „Natürlich“ bedeutet also paradoxerweise eine durch Gott gewollte gesellschaftliche Standortvergabe für die Person. Soll Ungleichheit abgeschafft werden, muss Geltung von transzendenten Mächten abgelöst und formalisiert werden. Jeder Einzelne ist eines unter einer Vielzahl von gesellschaftlichen Ereignissen der gleichen Art. Ausgehend vom Prinzip der Gleichheit wird die Gültigkeit von Tatbeständen so definiert, dass Individualität, d.h. der Einzelne in seiner Gültigkeit, formal gehandhabt werden kann.

Formal ist diese Gleichgültigkeit insofern, als keine Art von gesellschaftlicher oder natürlicher Inhaltsbestimmung wie etwa Reichtum, Stand, Beruf, Genialität, Körperkraft und -geschicklichkeit, Geschlecht, Wissen usw. einem Einzelnen Vorteile vor dem Gesetz gegenüber anderen verschaffen könnte. Das Besondere zählt nicht, so wie es auch im wissenschaftlichen Experiment ausgeschaltet wird. Das heißt: Weil der Maßstab der Beurteilung, das Gesetz, eine personenunabhängige, auf Übereinkunft beruhende Sammlung von Regeln ist, für die jedes Individuum in der Gesellschaft ein anonymes Einzelereignis darstellt, kann das Verhältnis von Individuum und Gesellschaft als formale Subsumtion unter das geltende Recht organisiert werden. Und es muss so organisiert werden, weil andernfalls keine Gleichbehandlung der Ereignisse vorläge. In diesem Sinne ist das Verfahren für alle objektiv, weil es intersubjektiv ist.

\section{Die Konterrevolution - und eine Zusammenfassung}

Dieser politische Exkurs sollte begründen, warum es vernünftig ist, die experimentellen Erfahrungswissenschaften demokratisch zu nennen. Sie funktionieren nach dem gleichen Prinzip (Eisel, 2006). Dann gilt das gleiche für die moderne Geographie im Verhältnis zur klassischen, wenn der Paradigmenwechsel eine Transformation ins experimentalwissenschaftliche Methodenideal bedeutete. William Warntz hat 1964 daher die These vertreten, dass die Sozialphysik und der spatial approach eine Wendung der Geographie zur Demokratie hin bedeute (Warntz, 1964).

Wir benötigen diesen Zusammenhang, um die dritte Phase der Revolution zu verstehen. In dieser Phase ereignete sich eine merkwürdige Widersprüchlichkeit. In der „humanistic geography“ wurde der behavioral approach bestätigt, aber zugleich wieder an das klassische Paradigma angeschlossen. Man kehrte zur idiographischen Weltsicht zurück, blieb aber - mehr oder weniger erfolgreich - bei der Abkehr von der klassischen Landschafts- und Länderkunde. Die Protagonisten beriefen sich auf die phänomenologische Philosophie und klagten de facto wieder das idiographische Methodenideal ein. Das war natürlich kein Zufall, denn diese Art der modernisierten Rehabilitation des zerstörten klassisch-geographischen Paradigmas setzte konsequenterweise unbewusst bei dessen inhaltlichem Kern an: dem - oben charakterisierten - humanistischen Individualitätsbegriff. Husserl hat sich ja explizit - so z. B. in den Pariser Vorträgen von 1929 oder in den Cartesianischen Meditationen auf die Monadologie von Leibniz berufen, in der die humanistische Idee der Individualität systemtheoretisch begründet wird; Husserl nennt seine Philosophie ,transzendentalphänomenologische Monadologie“ (Husserl, 1963:36).

Als erscheinendes Bewusstsein ist das klassische geographische Paradigma die Negation der industriekapitalistischen Produktionsweise zum Zeitpunkt ihrer Entstehung und Ausbreitung durch den Weltmarkt. Der Vorgang hat eine allgemeine Ebene, in welche die Geographie eingebunden ist. Die neuen Produktionsverhältnisse haben eine demokratische Staatsform zur Folge und sind mit dem Fortschrittsprinzip verbunden. Diese neuen ökonomischen und politischen Prinzipien konstituieren den Sinn ihrer politischen Vorgänger. Das bedeutet, dass deren Position als neue erst in diesem Sinnbezug entsteht, obwohl sie als eigenständige Weltdeutung längst existierte. Diese Gegenposition zu den neuen Prinzipien begründet sich in ihrer ursprünglichen Herkunft durch die humanistische Idee der Individualität. Das ist das, was schon vorlag als eine Weltauffassung, nun aber als Geisteshaltung der neuen modernen Welt entgegenstand. Andererseits wird diese Haltung als humanistische Idee von Menschenwürde gerade erst mit dem Sieg des Neuen ausformuliert im modernen Bewusstsein und damit auch in der Geographie. Sie besaß bis dahin noch unabhängig von der neuen Produktionsweise ihren Sinnhorizont im Individuali- 
tätsbegriff der Antike und des Christentums. Diese Traditionen geraten nun in den Deutungszusammenhang der Idee des Fortschritts, die den Kapitalverwertungsprozess als technisch verbesserte Anpassung an die Natur heiligspricht.

Mit diesem neuen Deutungszusammenhang wird aus dem Humanismus auf einer parallellaufenden aber anderen Ebene die politische Haltung des Konservatismus. Das alte geographische Paradigma in seiner Negation der Abstraktionsprinzipien der Industrie entspricht dieser Haltung. Davon ist nicht berührt, ob und wie die Geographen sich fachpolitisch mit der neuen Welt arrangierten. Sie begrüßten sie zumeist und muteten damit ihrem Paradigma einiges zu. Eine der Standardlösungen des entstehenden Dilemmas, in der sie eine rassische und völkische Besonderheit als historische Berufung zur industriellen Produktionsweise idiographisch zu deuten versuchten, habe ich oben angedeutet.

Berücksichtigt man die Zusammenhänge zwischen der Struktur der industriekapitalistischen Produktionsweise und den ihr entsprechenden bzw. widersprechenden Begriffen von Individualität, so ergeben sich folgende Ideen vom Subjekt als das Paradigma wechselt: Die „Humanistische Geographie" ist das erscheinende Bewusstsein des konservativen Bürgertums mit einem humanistischen Individualitätsbegriff und die wissenschaftstheoretisch modernisierte, arrivierte Form des alten Paradigmas. Dem ging im spatial approach, entsprechend der Idee der Einheit der Welt in abstrakter Natur aus der Sozialphysik, das erscheinende Bewusstsein der Produktionsweise überhaupt voraus. Das Subjekt des ökonomischen Prozesses ist das Kapital. Es umfasst als Anpassungsprinzip auf der ökonomischen Wertebene die Naturkraft der Arbeit und die Rohstoffe im sogenannten Mensch-Maschine-System der industriekapitalistischen Produktionsweise. Das menschliche Arbeitssubjekt ereignet sich gewissermaßen systemisch, nämlich als abstrakt objektivierte Systemeinheit des Mensch-Maschine-Systems - so wie in der Ergonomie beschrieben.

Im nachfolgenden behavioral approach erschien das Bewusstsein des demokratischen Bürgertums mit seinem Begriff abstrakter Individualität.

Die beiden vom Raumbegriff befreiten approaches, die humanistische Geographie und der behavioral approach, reflektieren das gesellschaftliche Subjekt kulturell und politisch, nämlich sowohl den humanistischen Individualitätsbegriff in seiner modernen politischen Form als auch den demokratischen Einzelnen; der approach des abstrakten Raums dagegen, der spatial approach, enthält noch den Blick auf die Naturanpassung und ihre Kapitalform - dies aber natürlich nur als objektivistische Spiegelung des Abstraktionsgrades dieser Systemeinheit in den räumlichen Effekten der Industrie, also im räumlichen Blick von außen auf die Standort- und Bewegungsmuster des Mensch-Natur-Verhältnisses.

Das ist meine Erklärung der Quantitativen Revolution.
Interessenkonflikt. Der Autor erklärt, dass kein Interessenkonflikt besteht.

Edited by: B. Korf

Reviewed by: two anonymous referees

\section{Literatur}

Althusser, L., Balibar, É., Establet, R., Macherey, P., und Rancière, J.: Das Kapital lesen, Münster, 2015.

Bunge, W.: Theoretical Geography, Lund Studies in Geography, Ser. C. General and Mathematical Geography, No. 1, 1966.

Dörrenhaus, F.: Geographie ohne Landschaft? Zu einem Aufsatz von Gerhard Hard, Geogr. Z., 59, 101-116, 1971.

Eisel, U.: Die Entwicklung der Anthropogeographie von einer „Raumwissenschaft“" zur Gesellschaftswissenschaft, Urbs et Regio, Kasseler Schriften zur Geografie und Planung, Band 17, Kassel, 1980.

Eisel, U.: Die Struktur politischer Geltung des Bürgers und die Struktur der Erfahrungswissenschaft. Teil I, in: Eisel, U., Körner, S., Die Versachlichung der Welt. Über die Rolle der Wissenschaft in der Demokratie, in: Landschaft in einer Kultur der Nachhaltigkeit, Band 1. Die Verwissenschaftlichung kultureller Qualität. Arbeitsberichte des Fachbereichs Architektur Stadtplanung Landschaftsplanung, Herausgeber: Eisel, U. und Körner, S., Heft 163, Universität Kassel, Kassel, S. 8-17, Uni-Bibliothek Kassel, 2006.

Eisel, U.: Die Hintergründe des Raumes. Eine Einführung in die Verflechtung von einigen Gedanken, 15-40, 2009a.

Eisel, U.: Landschaft und Gesellschaft. Räumliches Denken im Visier, Herausgeber: Belina, B., Michel, B., und Wissen, M., Raumproduktionen: Theorie \& gesellschaftliche Praxis, Band 5, Münster, 308 Seiten, 2009b.

Engels, F.: Briefe über materialistische Geschichtsinterpretation: Briefe an J. Bloch und F. Mehring, in: Karl Marx, Friedrich Engels, Herausgeber: Fetscher, I., Studienausgabe in 4 Bänden, Bd. I, Frankfurt/M., 1966.

Geografiker: Hefte 3-7/8 (1969-1972), Berlin, 226-235, 1966.

Hägerstrand, T.: Innovation Diffusion as a spatial process, The University of Chicago Press, Chicago/London, 1967.

Hard, G.: Was ist eine Landschaft? Über Etymologie als Denkform in der geographischen Literatur, in: Wirtschafts- und Sozialgeographie, Herausgeber: Bartels, D., Köln und Berlin, 171-197, 1970.

Hard, G.: Ärger mit Kurven (Zu F. Dörrenhaus: Geographie ohne Landschaft. Zu einem Aufsatz von Gerhard Hard), Geogr. Z., 59, 277-289, 1971.

Hard, G.: Antwort, Geografiker 7/8, Berlin, 47-52, 1972.

Hard, G.: Für eine konkrete Wissenschaftskritik, in: Wissenschaft und Wirklichkeit, Herausgeber: Anderegg, J., Göttingen, 134$161,1977$.

Hard, G.: Über die Gleichzeitigkeit des Ungleichzeitigen. Anmerkungen zur jüngsten methodologischen Literatur in der deutschen Geographie, in: Landschaft und Raum. Aufsätze zur Theorie der Geographie, Herausgeber: Hard, G., Band 1, Osnabrück, 133-154 (zuerst in Geografiker 6, 1971, Berlin, 12-24), 2002.

Husserl, E.: Husserliana. Edmund Husserl, Gesammelte Werke, Bd 1, Cartesianische Meditiationen und Pariser Vorträge, Herausgegeben und eingeleitet von Strasser, S., Haag, 1963. 
Laurig, W. und Rohmert, W.: Ergonomische Methoden zur Beurteilung des Teilsystems „Mensch“ in Arbeitssystemen, in: Ergonomie, Bd. 2, Herausgeber: Schmidtke, H., München, 113-145, 1974.

Kraus, T.: Individuelle Länderkunde, in: Probleme der Länderkunde, Herausgeber: Stewig, R., Darmstadt, 112-117, 1979.

Otremba, E.: Allgemeine Agrar- und Industriegeographie, in: Erde und Weltwirtschaft, Band 3, Herausgeber: Lüthgens, R., Stuttgart, 1960.

Ratzel, F.: Anthropo-Geographie oder Grundzüge der Anwendung der Erdkunde auf die Geschichte, Stuttgart, 1882.

Schlüter, O.: Die leitenden Gesichtspunkte der Anthropogeographie, insbesondere der Lehre Friedrich Ratzels, Archiv für Sozialwissenschaft und Sozialpolitik, 22, 581-630, 1906.

Schwind, M.: Kulturlandschaft als objektivierter Geist, Deutsche Geogr. Blätter, 46, Geogr. Ges. Bremen, 5-28, 1951.

Stewart, J. Q.: The development of social physics, Am. J. Phys., 18, 239-253, 1950.

Stewart, J. Q.: Social physics and the constitution of the United States, Address to the Foundation for Integrated Education, Philadelphia, 1953.

Stewart, J. Q. and Warntz, W. Macrogeography and social science, The Geographical Review, 48, 167-184, 1958.

Stewart, J. Q. and Warntz, W.: Physics of population distribution, in: Spatial analysis, Englewood Cliffs, edited by: Berry, B. J. L. and Marble, D. F., 130-146, 1968.
Stewig, R. (Hrsg.): Probleme der Länderkunde, Darmstadt, 1979.

Warntz, W.: The unity of knowledge, social science, and the role of geography, Rede bei der geographischen Sitzung des National Council for the Social Studies, Pittsburgh, 1957a.

Warntz, W.: Transportation, social physics, and the law of transportation, The Professional Geographer, 9, 2-7, $1957 \mathrm{~b}$.

Warntz, W.: New geography as general systems theory - old social physics writ large?, in: Directions in Geography, edited by: Corley, R. J., London, 89-126, 1973.

Warntz, W.: Geography now and then, American Geographical Society, Research Series 25, New York, 1964.

Werlen, B.: Gesellschaft, Handlung und Raum. Grundlagen handlungstheoretischer Sozialgeographie, Stuttgart, 1987.

Werlen, B.: Sozialgeographie alltäglicher Regionalisierungen, Bd. 2, Globalisierung, Region und Regionalisierung, Stuttgart, 1997.

Windelband, W.: Geschichte und Naturwissenschaft, Straßburger Rektoratsrede, in: Präludien. Aufsätze und Reden zur Philosophie und ihrer Geschichte, Zweiter Band, Tübingen, 136-160, (zuerst 1894), 1924.

Wirth, E.: Offener Brief an Herrn Prof. Dr. G. Hard, Geografiker, 7/8, Berlin, 45-46, 1972.

Wolpert, J.: Eine räumliche Analyse des Entscheidungsverhaltens in der mittelschwedischen Landwirtschaft, in: Wirtschafts- und Sozialgeographie, Herausgeber: Bartels, D., Köln/Berlin, 380387 (zuerst 1967), 1970. 